\title{
Deconfinement transition in protoneutron stars: analysis within the Nambu-Jona-Lasinio model
}

\author{
G. Lugones ${ }^{1}$, T. A. S. do Carmo ${ }^{1}$, A. G. Grunfeld ${ }^{2,3,4}$, N. N. Scoccola ${ }^{2,3,5}$ \\ 1 Universidade Federal do ABC, Centro de Ciencias Naturais e Humanas, \\ Rua Santa Adélia, 166, 09210-170, Santo André, Brazil \\ 2 CONICET, Rivadavia 1917, (1033) Buenos Aires, Argentina. \\ ${ }^{3}$ Departmento de Física, Comisión Nacional de Energía Atómica, (1429) Buenos Aires, Argentina. \\ 4 Department of Physics, Sultan Qaboos University, \\ P.O.Box: 36 Al-Khode 123 Muscat, Sultanate of Oman \\ 5 Universidad Favaloro, Solís 453, (1078) Buenos Aires, Argentina.
}

\begin{abstract}
We study the effect of color superconductivity and neutrino trapping on the deconfinement transition of hadronic matter into quark matter in a protoneutron star. To describe the strongly interacting matter a two-phase picture is adopted. For the hadronic phase we use different parameterizations of a non-linear Walecka model which includes the whole baryon octet. For the quark matter phase we use an $S U(3)_{f}$ Nambu-Jona-Lasinio effective model which includes color superconductivity. We impose color and flavor conservation during the transition in such a way that just deconfined quark matter is transitorily out of equilibrium with respect to weak interactions. We find that deconfinement is more difficult for small neutrino content and it is easier for lower temperatures although these effects are not too large. In addition they will tend to cancel each other as the protoneutron star cools and deleptonizes, resulting a transition density that is roughly constant along the evolution of the protoneutron star. According to these results the deconfinement transition is favored after substantial cooling and contraction of the protoneutron star.
\end{abstract}

PACS numbers: $12.39 . \mathrm{Fe}, 25.75 . \mathrm{Nq}, 26.60 . \mathrm{Kp}$

\section{INTRODUCTION}

It is currently a matter of speculation the actual occurrence of quark matter during protoneutron star (PNS) evolution. The standard scenario for the birth of neutron stars indicates that these objects are formed as consequence of the gravitational collapse and supernova explosion of a massive star [1 $\left[\begin{array}{l}3 \\ ]\end{array}\right.$. Initially, PNSs are very hot and leptonrich objects, where neutrinos are temporarily trapped. During the first tens of seconds of evolution the PNS evolves to form a cold $\left(T<10^{10} \mathrm{~K}\right)$ catalyzed neutron star [1-3]. As neutrinos are radiated, the lepton - per - baryon content of matter goes down and the neutrino chemical potential tends to essentially zero in $\sim 50$ seconds [3]. Deleptonization is fundamental for quark matter formation inside neutron stars, since it has been shown that the presence of trapped neutrinos in hadronic matter strongly disfavors the deconfinement transition [4, 5]. In fact, neutrino trapping makes the density for the deconfinement transition to be higher than in the case of neutrino-free hadronic matter. As a consequence, the transition could be delayed several seconds after the bounce of the stellar core. However, the calculations presented in [4, 5] were performed employing the MIT Bag model for the description of quark matter and did not include the effect of color superconductivity. As we shall see in the present work, the use of the Nambu-JonaLasinio model and the inclusion of color superconductivity may change qualitatively the effect of neutrino trapping in the deconfinement conditions.

As emphasized in earlier works [4 11], an important characteristic of the deconfinement transition in neutron stars, is that just deconfined quark matter is transitorily out of equilibrium with respect to weak interactions. In fact, depending on the temperature, the transition should begin with the quantum or thermal nucleation of a small quarkmatter drop near the center of the star. On the other hand, the flavor composition of hadronic matter in $\beta$-equilibrium is different from that of a $\beta$-stable quark-matter drop. Roughly speaking, the direct formation of a $\beta$-stable quark-drop with $N$ quarks will need the almost simultaneous conversion of $\sim N / 3$ up and down quarks into strange quarks, a process which is strongly suppressed with respect to the formation of a non $\beta$-stable drop by a factor $\sim G_{\mathrm{Fermi}}^{2 N / 3}$. For typical values of the critical-size $\beta$-stable drop $(N \sim 100-1000[6])$ the suppression factor is actually tiny. Thus, quark flavor must be conserved during the deconfinement transition [4 10]. When color superconductivity is included together with flavor conservation, the most likely configuration of the just deconfined phase is 2SC provided the pairing gap is large enough [9]. The relevance of this 2SC intermediate phase (a kind of activation barrier) has been analyzed for deleptonized neutron stars [10, 12] but not for hot and lepton-rich objects like PNSs.

In the present paper we shall analyze the deconfinement transition in protoneutron star conditions employing the Nambu-Jona-Lasinio model in the description of quark matter. For the hadronic phase we shall use a model based on a relativistic Lagrangian of hadrons interacting via the exchange of $\sigma, \rho$, and $\omega$ mesons [13]. For simplicity, the analysis will be made in bulk, i.e. without taking into account the energy cost due to finite size effects in creating a 


\begin{tabular}{c|c|c|c|c|c|c|c|c|c}
\hline \hline Label & composition & $x_{\sigma}=x_{\rho}$ & $x_{\omega}$ & $\begin{array}{c}\left(g_{\sigma} / m_{\sigma}\right)^{2} \\
{\left[\mathrm{fm}^{2}\right]}\end{array}$ & $\begin{array}{c}\left(g_{\omega} / m_{\omega}\right)^{2} \\
{\left[\mathrm{fm}^{2}\right]}\end{array}$ & $\begin{array}{c}\left(g_{\rho} / m_{\rho}\right)^{2} \\
{\left[\mathrm{fm}^{2}\right]}\end{array}$ & $b$ & $c$ & $M_{\max }$ \\
\hline GM 1 & baryon octet $+e^{-}$ & 0.6 & 0.653 & 11.79 & 7.149 & 4.411 & 0.002947 & -0.001070 & $1.78 M_{\odot}$ \\
GM 4 & baryon octet $+e^{-}$ & 0.9 & 0.9 & 11.79 & 7.149 & 4.411 & 0.002947 & -0.001070 & $2.2 M_{\odot}$ \\
\hline \hline
\end{tabular}

TABLE I: Parameters of the hadronic equation of state. For each parametrization we give the maximum mass $M_{m a x}$ of a hadronic star.

drop of deconfined quark matter in the hadronic environment.

The present article is organized as follows. In Sec. II we present the main aspects of the non-linear Walecka model describing the hadronic phase. In Sec. III we present the generalities of the model we use for the quark phase. In Sec. IV we show our numerical results and finally in Sec. V we discuss our results and present the conclusions.

\section{THE HADRONIC PHASE}

For the hadronic phase we shall use a non-linear Walecka model (NLWM) [13 15] which includes the whole baryon octet, electrons and electron neutrinos in equilibrium under weak interactions. The Lagrangian of the model is given by

$$
\mathcal{L}=\mathcal{L}_{B}+\mathcal{L}_{M}+\mathcal{L}_{L}
$$

where the indices $B, M$ and $L$ refer to baryons, mesons and leptons respectively. For the baryons we have

$$
\mathcal{L}_{B}=\sum_{B} \bar{\psi}_{B}\left[\gamma^{\mu}\left(i \partial_{\mu}-g_{\omega B} \omega_{\mu}-g_{\rho B} \vec{\tau} \cdot \vec{\rho}_{\mu}\right)-\left(m_{B}-g_{\sigma B} \sigma\right)\right] \psi_{B}
$$

with $B=n, p, \Lambda, \Sigma^{+}, \Sigma^{0}, \Sigma^{-}, \Xi^{-}$, and $\Xi^{0}$. The contribution of the mesons $\sigma, \omega$ and $\rho$ is given by

$$
\begin{aligned}
\mathcal{L}_{M}= & \frac{1}{2}\left(\partial_{\mu} \sigma \partial^{\mu} \sigma-m_{\sigma}^{2} \sigma^{2}\right)-\frac{b}{3} m_{N}\left(g_{\sigma} \sigma\right)^{3}-\frac{c}{4}\left(g_{\sigma} \sigma\right)^{4} \\
& -\frac{1}{4} \omega_{\mu \nu} \omega^{\mu \nu}+\frac{1}{2} m_{\omega}^{2} \omega_{\mu} \omega^{\mu}-\frac{1}{4} \vec{\rho}_{\mu \nu} \cdot \vec{\rho}^{\mu \nu}+\frac{1}{2} m_{\rho}^{2} \vec{\rho}_{\mu} \cdot \vec{\rho}^{\mu},
\end{aligned}
$$

where the coupling constants are $g_{\sigma B}=x_{\sigma B} g_{\sigma}, g_{\omega B}=x_{\omega B} g_{\omega}$ and $g_{\rho B}=x_{\rho B} g_{\rho}$. The ratios $x_{\sigma B}, x_{\omega B}$ and $x_{\rho B}$ are equal to 1 for the nucleons and acquire different values for the other baryons depending on the parametrization (see Table I). The leptonic sector is included as a free Fermi gas of electrons and electron neutrinos in chemical equilibrium with the other particles.

There are five constants in the model that are determined by the properties of nuclear matter, three that determine the nucleon couplings to the scalar, vector and vector-isovector mesons $g_{\sigma} / m_{\sigma}, g_{\omega} / m_{\omega}, g_{\rho} / m_{\rho}$, and two that determine the scalar self interactions $b$ and $c$. It is assumed that all hyperons in the octet have the same coupling than the $\Lambda$. These couplings are expressed as a ratio to the nucleon couplings mentioned above, that we thus simply denote $x_{\sigma}, x_{\omega}$ and $x_{\rho}$. In the present work we use two parameterizations for the constants. One of them is the standard parameterization GM1 given by Glendenning-Moszkowski [13], as shown in Table I. This parametrization employs "low" values for $x_{\sigma}, x_{\omega}$ and $x_{\rho}$. The parametrization GM4 employs larger values of these couplings. This makes the EOS stiffer and increases the maximum mass of hadronic stars to $2.2 M_{\odot}$, see Table I.

The derivation of the equations describing the model is given in detail in [16]. The total pressure $P$ and mass energy density $\rho$ are given by:

$$
\begin{gathered}
P=\sum_{i=B, L} P_{i}+\frac{1}{2}\left(\frac{g_{\omega}}{m_{\omega}}\right)^{2} \rho_{B}^{\prime 2}-\frac{1}{2}\left(\frac{g_{\sigma}}{m_{\sigma}}\right)^{-2}\left(g_{\sigma} \sigma\right)^{2}-\frac{1}{3} b m_{n}\left(g_{\sigma} \sigma\right)^{3}-\frac{1}{4} c\left(g_{\sigma} \sigma\right)^{4}+\frac{1}{2}\left(\frac{g_{\rho}}{m_{\rho}}\right)^{2} \rho_{I_{3}}^{\prime 2}, \\
\rho=\sum_{i=B, L} \rho_{i}+\frac{1}{2}\left(\frac{g_{\omega}}{m_{\omega}}\right)^{2} \rho_{B}^{\prime 2}+\frac{1}{2}\left(\frac{g_{\sigma}}{m_{\sigma}}\right)^{-2}\left(g_{\sigma} \sigma\right)^{2}+\frac{1}{3} b m_{n}\left(g_{\sigma} \sigma\right)^{3}+\frac{1}{4} c\left(g_{\sigma} \sigma\right)^{4}+\frac{1}{2}\left(\frac{g_{\rho}}{m_{\rho}}\right)^{2} \rho_{I_{3}}^{\prime 2} .
\end{gathered}
$$

Here $P_{i}$ and $\rho_{i}$ are the expressions for a Fermi gas of relativistic, non-interacting particles:

$$
P_{i}=\frac{1}{3} \frac{g_{i}}{(2 \pi)^{3}} \int d^{3} p \frac{p^{2}}{\left(p^{2}+m_{i}^{* 2}\right)^{1 / 2}}\left(f_{i}(T)+\bar{f}_{i}(T)\right)
$$




$$
\rho_{i}=\frac{g_{i}}{(2 \pi)^{3}} \int d^{3} p\left(p^{2}+m_{i}^{* 2}\right)^{1 / 2}\left(f_{i}(T)+\bar{f}_{i}(T)\right)
$$

where $f_{i}(T)$ and $\bar{f}_{i}(T)$ are the Fermi - Dirac distribution functions for particles and antiparticles respectively:

$$
\begin{aligned}
& f_{i}(T)=\left(\exp \left(\left[\left(p^{2}+m_{i}^{* 2}\right)^{1 / 2}-\mu_{i}^{*}\right] / T\right)+1\right)^{-1} \\
& \bar{f}_{i}(T)=\left(\exp \left(\left[\left(p^{2}+m_{i}^{* 2}\right)^{1 / 2}+\mu_{i}^{*}\right] / T\right)+1\right)^{-1} .
\end{aligned}
$$

Note that for baryons we use, instead of masses $m_{i}$ and chemical potentials $\mu_{i}$, "effective" masses $m_{i}^{*}$ and chemical potentials $\mu_{i}^{*}$ given by:

$$
\begin{gathered}
m_{i}^{*}=m_{i}+x_{\sigma i}\left(g_{\sigma} \sigma\right) \\
\mu_{i}^{*}=\mu_{i}-x_{\omega i}\left(\frac{g_{\omega}}{m_{\omega}}\right)^{2} \rho_{B}^{\prime}-x_{\rho i} I_{3 i}\left(\frac{g_{\rho}}{m_{\rho}}\right)^{2} \rho_{I_{3}}^{\prime},
\end{gathered}
$$

where $I_{3 i}$ is the third component of the isospin of each baryon.

The weighted isospin density $\rho_{I_{3}}^{\prime}$ and the weighted baryon density $\rho_{B}^{\prime}$ are given by:

$$
\begin{gathered}
\rho_{I_{3}}^{\prime}=\sum_{i=B} x_{\rho i} I_{3 i} n_{i}, \\
\rho_{B}^{\prime}=\sum_{i=B} x_{\omega i} n_{i},
\end{gathered}
$$

being $n_{i}$ the particle number density of each baryon:

$$
n_{i}=\frac{g_{i}}{(2 \pi)^{3}} \int d^{3} p\left(f_{i}(T)-\bar{f}_{i}(T)\right) .
$$

The mean field $g_{\sigma} \sigma$ satisfies the equation:

$$
\left(\frac{g_{\sigma}}{m_{\sigma}}\right)^{-2}\left(g_{\sigma} \sigma\right)+b m_{n}\left(g_{\sigma} \sigma\right)^{2}+c\left(g_{\sigma} \sigma\right)^{3}=\sum_{i=B} x_{\sigma i} n_{i}^{s}
$$

where $n_{i}^{s}$ is the scalar density:

$$
n_{i}^{s}=\frac{g_{i}}{(2 \pi)^{3}} \int d^{3} p \frac{m_{i}^{*}}{\left(p^{2}+m_{i}^{* 2}\right)^{1 / 2}}\left(f_{i}(T)+\bar{f}_{i}(T)\right) .
$$

The hadron phase is assumed to be charge neutral and in chemical equilibrium under weak interactions. Electric charge neutrality states:

$$
n_{p}+n_{\Sigma^{+}}-n_{\Sigma^{-}}-n_{\Xi^{-}}-n_{e}=0
$$

Chemical weak equilibrium in the presence of trapped electron neutrinos implies that the chemical potential $\mu_{i}$ of each baryon in the hadron phase is given by:

$$
\mu_{i}=q_{B} \mu_{n}-q_{e}\left(\mu_{e}-\mu_{\nu_{e}}\right)
$$

where $q_{B}$ is its baryon charge and $q_{e}$ is its electric charge. For simplicity we are assuming that muon and tau neutrinos are not present in the system, and their chemical potentials are set to zero.

All the above equations can be solved numerically by specifying three thermodynamic quantities, e.g. the temperature $T$, the mass-energy density $\rho$ and the chemical potential of electron neutrinos in the hadronic phase $\mu_{\nu_{e}}^{H}$. 


\section{THE QUARK MATTER PHASE}

In order to study the just deconfined quark matter phase we use an $S U(3)_{f}$ NJL effective model which also includes color superconducting quark-quark interactions. The corresponding Lagrangian is given by

$$
\mathcal{L}=\bar{\psi}(i \not \partial-\hat{m}) \psi+G \sum_{a=0}^{8}\left[\left(\bar{\psi} \tau_{a} \psi\right)^{2}+\left(\bar{\psi} i \gamma_{5} \tau_{a} \psi\right)^{2}\right]+2 H \sum_{A, A^{\prime}=2,5,7}\left[\left(\bar{\psi} i \gamma_{5} \tau_{A} \lambda_{A^{\prime}} \psi_{C}\right)\left(\bar{\psi}_{C} i \gamma_{5} \tau_{A} \lambda_{A^{\prime}} \psi\right)\right]
$$

where $\hat{m}=\operatorname{diag}\left(m_{u}, m_{d}, m_{s}\right)$ is the current mass matrix in flavor space. In what follows we will work in the isospin symmetric limit $m_{u}=m_{d}=m$. Moreover, $\tau_{i}$ and $\lambda_{i}$ with $i=1, . ., 8$ are the Gell-Mann matrices corresponding to the flavor and color groups respectively, and $\tau_{0}=\sqrt{2 / 3} 1_{f}$. Finally, the charge conjugate spinors are defined as follows: $\psi_{C}=C \bar{\psi}^{T}$ and $\bar{\psi}_{C}=\psi^{T} C$, where $\bar{\psi}=\psi^{\dagger} \gamma^{0}$ is the Dirac conjugate spinor and $C=i \gamma^{2} \gamma^{0}$.

To be able to determine the relevant thermodynamical quantities we have to obtain the grand canonical thermodynamical potential at finite temperature $T$ and chemical potentials $\mu_{f c}$. Here, $f=(u, d, s)$ and $c=(r, g, b)$ denotes flavor and color indices respectively. For this purpose, starting from Eq. (19), we perform the usual bosonization of the theory. This can be done by introducing scalar and pseudoscalar meson fields $\sigma_{a}$ and $\pi_{a}$ respectively, together with the bosonic diquark field $\Delta_{A}$. In this work we consider the quantities obtained within the mean field approximation (MFA). Thus, we only keep the non-vanishing vacuum expectation values of these fields and drop the corresponding fluctuations. For the meson fields this implies $\hat{\sigma}=\sigma_{a} \tau_{a}=\operatorname{diag}\left(\sigma_{u}, \sigma_{d}, \sigma_{s}\right)$ and $\pi_{a}=0$. Concerning the diquark mean field, we will assume that in the density region of interest only the 2SC phase might be relevant. Thus, we adopt the ansatz $\Delta_{5}=\Delta_{7}=0, \Delta_{2}=\Delta$. Integrating out the quark fields and working in the framework of the Matsubara and Nambu-Gorkov formalism we obtain the following MFA quark thermodynamical potential (a detailed procedure of calculation can be found in Refs. [17 19] )

$$
\Omega_{q}^{M F A}\left(T, \mu_{f c}, \sigma_{u}, \sigma_{d}, \sigma_{s},|\Delta|\right)=\frac{1}{\pi^{2}} \int_{0}^{\Lambda} d k k^{2} \sum_{i=1}^{9} \omega\left(x_{i}, y_{i}\right)+\frac{1}{4 G}\left(\sigma_{u}^{2}+\sigma_{d}^{2}+\sigma_{s}^{2}\right)+\frac{|\Delta|^{2}}{2 H},
$$

where $\Lambda$ is the cut-off of the model and $\omega(x, y)$ is defined by

$$
\omega(x, y)=-\left[x+T \ln \left[1+e^{-(x-y) / T}\right]+T \ln \left[1+e^{-(x+y) / T}\right]\right],
$$

with

$$
\begin{gathered}
x_{1,2}=E \quad, \quad x_{3,4,5}=E_{s} \quad, \quad x_{6,7}=\sqrt{\left[E+\frac{\left(\mu_{u r} \pm \mu_{d g}\right)}{2}\right]^{2}+\Delta^{2}}, \quad x_{8,9}=\sqrt{\left[E+\frac{\left(\mu_{u g} \pm \mu_{d r}\right)}{2}\right]^{2}+\Delta^{2}}, \\
y_{1}=\mu_{u b}, \quad y_{2}=\mu_{d b}, \quad y_{3}=\mu_{s r}, \quad y_{4}=\mu_{s g}, \quad y_{5}=\mu_{s b}, \quad y_{6,7}=\frac{\left(\mu_{u r}-\mu_{d g}\right)}{2}, \quad y_{8,9}=\frac{\mu_{u g}-\mu_{d r}}{2} .
\end{gathered}
$$

Here, $E=\sqrt{k^{2}+M^{2}}$ and $E_{s}=\sqrt{k^{2}+M_{s}^{2}}$, where $M_{f}=m_{f}+\sigma_{f}$. Note that in the isospin limit we are working $\sigma_{u}=\sigma_{d}=\sigma$ and, thus, $M_{u}=M_{d}=M$.

The total thermodynamical potential of the quark matter phase (QMP) is obtained by adding to $\Omega_{M F A}$ the contribution of the leptons. Namely,

$$
\Omega_{Q M P}\left(T, \mu_{f c}, \mu_{e}, \mu_{\nu_{e}}, \sigma, \sigma_{s},|\Delta|\right)=\Omega_{q}^{M F A}\left(T, \mu_{f c}, \sigma, \sigma_{s},|\Delta|\right)+\Omega_{e}\left(T, \mu_{e}\right)+\Omega_{\nu_{e}}\left(T, \mu_{\nu_{e}}\right)-\Omega_{\text {vac }}
$$

where $\Omega_{e}$ and $\Omega_{\nu_{e}}$ are the thermodynamical potentials of the electrons and neutrinos, respectively. For them we use the expression corresponding to a free gas of ultra-relativistic fermions

$$
\Omega_{l}\left(T, \mu_{l}\right)=-\gamma_{l}\left(\frac{\mu_{l}^{4}}{24 \pi^{2}}+\frac{\mu_{l}^{2} T^{2}}{12}+\frac{7 \pi^{2} T^{4}}{360}\right)
$$

where $l=e, \nu_{e}$ and the degeneracy factor is $\gamma_{e}=2$ for electrons and $\gamma_{\nu_{e}}=1$ for neutrinos. Notice that in Eq.(23) we have subtracted the constant $\Omega_{\mathrm{vac}}$ in order to have a vanishing pressure at vanishing temperature and chemical potentials.

From the grand thermodynamic potential $\Omega_{Q M P}$ we can readily obtain the pressure $P=-\Omega_{Q M P}$, the number density of quarks of each flavor and color $n_{f c}=-\partial \Omega_{Q M P} / \partial \mu_{f c}$, the number density of electrons $n_{e}=-\partial \Omega_{Q M P} / \partial \mu_{e}$, 


\begin{tabular}{l|ccccc}
\hline \hline & $m_{u, d}[\mathrm{Mev}]$ & $m_{s}[\mathrm{Mev}]$ & $\Lambda[\mathrm{Mev}]$ & $G \Lambda^{2}$ & $H / G$ \\
\hline set 1 & 5.5 & 112.0 & 602.3 & 4.638 & $3 / 4$ \\
set 2 & 5.5 & 110.05 & 631.4 & 4.370 & $3 / 4$ \\
\hline
\end{tabular}

TABLE II: The two sets of NJL parameters.

and the number density of electron neutrinos $n_{\nu_{e}}=-\partial \Omega_{Q M P} / \partial \mu_{\nu_{e}}$. The corresponding number densities of each flavor, $n_{f}$, and of each color, $n_{c}$, in the quark phase are given by $n_{f}=\sum_{c} n_{f c}$ and $n_{c}=\sum_{f} n_{f c}$ respectively. The baryon number density reads $n_{B}=\frac{1}{3} \sum_{f c} n_{f c}=\left(n_{u}+n_{d}+n_{s}\right) / 3$. Finally, the Gibbs free energy per baryon is

$$
g_{\text {quark }}=\frac{1}{n_{B}}\left(\sum_{f c} \mu_{f c} n_{f c}+\mu_{e} n_{e}+\mu_{\nu_{e}} n_{\nu_{e}}\right) .
$$

For the NJL model we use two sets of constants shown in Table II] The sets 1 and 2 were taken from [20] and [21] respectively, but without the 't Hooft flavor mixing interaction. The procedure, obtained from [22] is to keep $\Lambda$ and $m$ fixed, then tune the remaining parameters $G$ and $m_{s}$ in order to reproduce $M=367.6 \mathrm{MeV}$ and $M_{s}=549.5 \mathrm{MeV}$ at zero temperature and density. The resulting parameter sets are given in Table [I]

In order to derive a quark matter EOS from the above formulae it is necessary to impose a suitable number of conditions on the variables $\left\{\mu_{f c}\right\}, \mu_{e}, \mu_{\nu_{e}}, \sigma, \sigma_{s}$ and $\Delta$. Three of these conditions are consequences from the fact that the thermodynamically consistent solutions correspond to the stationary points of $\Omega$ with respect to $\sigma, \sigma_{s}$, and $\Delta$. Thus, we have

$$
\partial \Omega_{Q M P} / \partial \sigma=0 \quad, \quad \partial \Omega_{Q M P} / \partial \sigma_{s}=0 \quad, \quad \partial \Omega_{Q M P} / \partial|\Delta|=0 .
$$

To obtain the remaining conditions one must specify the physical situation in which one is interested in. As in previous works [4-10], we are dealing here with just deconfined quark matter that is temporarily out of chemical equilibrium under weak interactions. The appropriate condition in this case is flavor conservation between hadronic and deconfined quark matter. This can be written as

$$
Y_{f}^{H}=Y_{f}^{Q} \quad f=u, d, s, e, \nu_{e}
$$

being $Y_{f}^{H} \equiv n_{f}^{H} / n_{B}^{H}$ and $Y_{i}^{Q} \equiv n_{f}^{Q} / n_{B}^{Q}$ the abundances of each particle in the hadron and quark phase respectively. In other words, the just deconfined quark phase must have the same "flavor" composition than the $\beta$-stable hadronic phase from which it has been originated. Notice that, since the hadronic phase is assumed to be electrically neutral, flavor conservation ensures automatically the charge neutrality of the just deconfined quark phase. The conditions given in Eq. (27) can be combined to obtain

$$
n_{d}=\xi n_{u} \quad, \quad n_{s}=\eta n_{u} \quad, \quad n_{\nu_{e}}=\kappa n_{u} \quad, \quad 3 n_{e}=2 n_{u}-n_{d}-n_{s},
$$

where $n_{i}$ is the particle number density of the $i$-species in the quark phase. The quantities $\xi \equiv Y_{d}^{H} / Y_{u}^{H}, \eta \equiv Y_{s}^{H} / Y_{u}^{H}$ and $\kappa \equiv Y_{\nu_{e}}^{H} / Y_{u}^{H}$ are functions of the pressure and temperature, and they characterize the composition of the hadronic phase. These expressions are valid for any hadronic EOS. For hadronic matter containing $n, p, \Lambda, \Sigma^{+}, \Sigma^{0}, \Sigma^{-}, \Xi^{-}$ and $\Xi^{0}$, we have

$$
\begin{aligned}
\xi & =\frac{n_{p}+2 n_{n}+n_{\Lambda}+n_{\Sigma^{0}}+2 n_{\Sigma^{-}}+n_{\Xi^{-}}}{2 n_{p}+n_{n}+n_{\Lambda}+2 n_{\Sigma^{+}}+n_{\Sigma^{0}}+n_{\Xi^{0}}}, \\
\eta & =\frac{n_{\Lambda}+n_{\Sigma^{+}}+n_{\Sigma^{0}}+n_{\Sigma^{-}}+2 n_{\Xi^{0}}+2 n_{\Xi^{-}}}{2 n_{p}+n_{n}+n_{\Lambda}+2 n_{\Sigma^{+}}+n_{\Sigma^{0}}+n_{\Xi^{0}}}, \\
\kappa & =\frac{n_{\nu_{e}}^{H}}{2 n_{p}+n_{n}+n_{\Lambda}+2 n_{\Sigma^{+}}+n_{\Sigma^{0}}+n_{\Xi^{0}}} .
\end{aligned}
$$

Additionally, the deconfined phase must be locally colorless; thus it must be composed by an equal number of red, green and blue quarks

$$
n_{r}=n_{g}=n_{b} .
$$


Also, $u r, u g, d r$, and $d g$ pairing will happen provided that $|\Delta|$ is nonzero, leading to

$$
n_{u r}=n_{d g} \quad, \quad n_{u g}=n_{d r} .
$$

In order to have all Fermi levels at the same value, we consider [9]

$$
n_{u g}=n_{u r} \quad, \quad n_{s b}=n_{s r} .
$$

These two equations, together with Eqs. (32) and (33) imply that $n_{u r}=n_{u g}=n_{d r}=n_{d g}$ and $n_{s r}=n_{s g}=n_{s b}$ [9].

Finally, including the conditions Eqs.(26) we have 13 equations involving the 14 unknowns $\left(\sigma, \sigma_{s},|\Delta|, \mu_{e}, \mu_{\nu_{e}}\right.$ and $\left.\left\{\mu_{f c}\right\}\right)$. For given value of one of the chemical potentials (e.g. $\mu_{u r}$ ), the set of equations can be solved once the values of the parameters $\xi, \eta, \kappa$ and the temperature $T$ are given. Instead of $\mu_{u r}$, we can provide a value of the Gibbs free energy per baryon $g_{\text {quark }}$ or the pressure $P$ and solve simultaneously Eqs. (28)-(34) together with Eq. (26) in order to obtain $\sigma, \sigma_{s},|\Delta|, \mu_{e}, \mu_{\nu_{e}}$ and $\left\{\mu_{f c}\right\}$.

\section{DECONFINEMENT TRANSITION IN PROTO-NEUTRON STAR MATTER}

In order to determine the transition conditions, we apply the Gibbs criteria, i.e. we assume that deconfinement will occur when the pressure and Gibbs energy per baryon are the same for both hadronic matter and quark matter at a given common temperature. Thus, we have

$$
g^{H}=g^{Q}, \quad P^{H}=P^{Q}, \quad T^{H}=T^{Q},
$$

where the index $H$ refers to hadron matter and the index $Q$ to quark matter. According to these conditions (together with the equations of Sections 2 and 3), for a given temperature $T^{H}$ and neutrino chemical potential of the trapped neutrinos in the hadronic phase $\mu_{\nu_{e}}^{H}$, there is an unique pressure $P$ at which the deconfinement is possible. Instead of $P$, we may characterize the transition point by giving the Gibbs free energy per baryon $g$, or alternatively, the massenergy density of the hadronic phase $\rho_{H}$ (see Figs. 1-3). We emphasize that, according to the present description, $P$ and $g$ are the same in both the hadronic phase and the just deconfined phase. However, the mass-energy density $\rho_{H}$ and $\rho_{Q}$ at the transition point are different in general. Similarly, while the abundance $Y_{\nu_{e}}$ of neutrinos is the same in both the hadronic and just deconfined quark phases, the chemical potentials $\mu_{\nu_{e}}^{Q}$ and $\mu_{\nu_{e}}^{H}$ are different.

According to numerical simulations [1-3], during the first tens of seconds of evolution the protoneutron star cools from $T \sim 40 \mathrm{MeV}$ to temperatures below 2-4 MeV. In the same period, the chemical potential $\mu_{\nu_{e}}^{H}$ of the trapped neutrinos evolves from $\sim 200 \mathrm{MeV}$ to essentially zero. Thus, in order to consider typical PNS conditions we have solved Eqs. (35) together with the equations of Sections 2 and 3 for temperatures in the range $0-60 \mathrm{MeV}$ and $\mu_{\nu_{e}}^{H}$ in the range $0-200 \mathrm{MeV}$. The results are displayed in Figs. 1-3 for all the parameterizations of the equations of state given in previous sections.

In Fig. 1 we display the results for the GM1 parametrization of the hadronic EOS. In the left panel of Fig. 1 we show the Gibbs free energy density per baryon $g$ at which deconfinement occurs versus the temperature $T$ for three different values of the neutrino chemical potential in the hadronic phase $\left(\mu_{\nu_{e}}^{H}=0,100,200 \mathrm{MeV}\right)$. In the right panel the same results are shown but as a function of the mass-energy density of the hadronic phase (in units of the nuclear saturation density $\rho_{0}=2.7 \times 10^{14} \mathrm{~g} \mathrm{~cm}^{3}$ ). In both figures, if the thermodynamic state of hadronic matter (characterized e.g. by $T^{H}, \rho_{H}$ and $\mu_{\nu_{e}}^{H}$ ) lies to the left of the curve corresponding to the same $\mu_{\nu_{e}}^{H}$, then the deconfinement transition is not possible. In the right side region of a given curve the preferred phase is deconfined quark matter. Notice that the transition's Gibbs free energy is an increasing function of $\mu_{\nu_{e}}^{H}$. However, the transition density of the hadronic phase slightly decreases as $\mu_{\nu_{e}}^{H}$ increases. In Fig. 2 we display the results for the GM4 parametrization of the hadronic EOS. The results are qualitatively the same but the transition densities are smaller than those for GM1 by $\sim 30 \%$.

In Fig. 3 we show the behavior of the transition's density as a function of the chemical potential of trapped neutrinos $\mu_{\nu_{e}}^{H}$ for two specific temperatures $(T=2$ and $30 \mathrm{MeV})$. It is clearly seen that for a fixed temperature the effect of deleptonization is to inhibit the transition. This effect is not very large; at fixed temperature there is a slight increase by less than a $10 \%$ when $\mu_{\nu_{e}}^{H}$ falls from $200 \mathrm{MeV}$ to $0 \mathrm{MeV}$. On the other hand, the effect of cooling works in the opposite direction because pairing tends to help the transition and the gap increases as the temperature goes down. The effect of cooling is also small; at fixed $\mu_{\nu_{e}}^{H}$ there is a slight decrease of the transition density $\rho_{H}$ by less than a $10 \%$ when the temperature falls from $30 \mathrm{MeV}$ to $2 \mathrm{MeV}$. Both effects tend to cancel each other as the PNS cools and deleptonizes, resulting a transition density that is roughly constant along the evolution of the protoneutron star. 

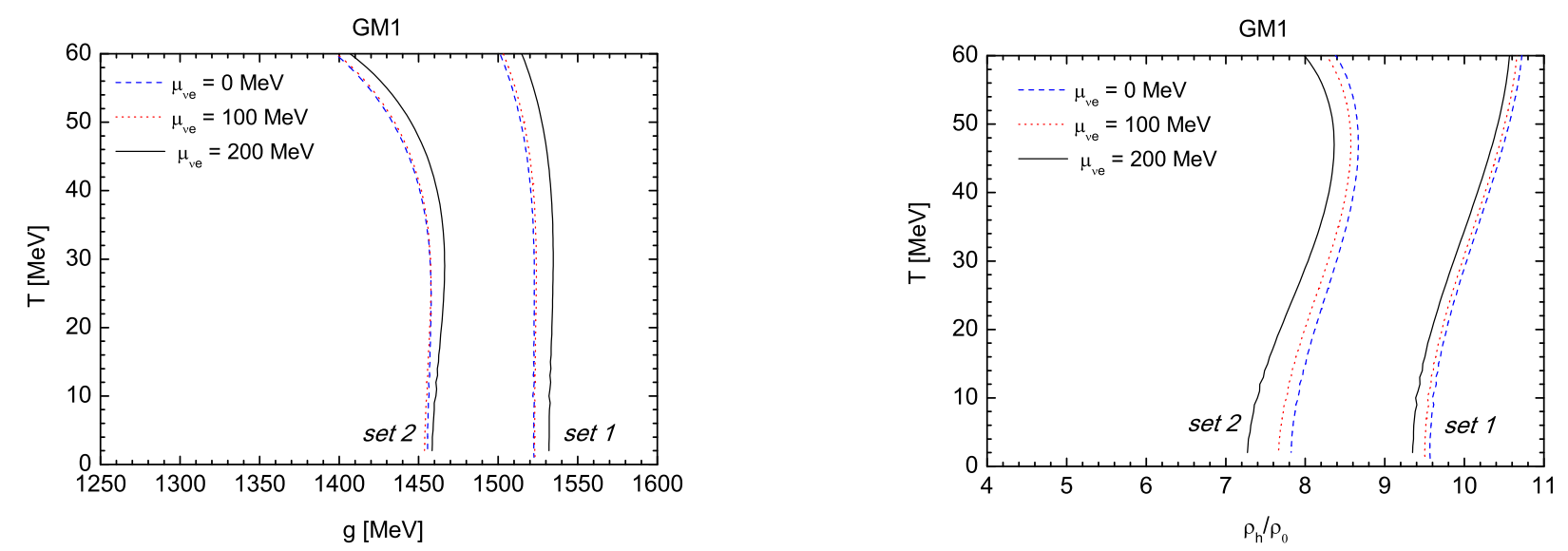

FIG. 1: Left panel: the Gibbs free energy density per baryon $g$ at which deconfinement occurs versus the temperature $T$ for three different values of the neutrino chemical potential in the hadronic phase $\left(\mu_{\nu_{e}}^{H}=0 \mathrm{MeV}\right.$ in dashed line, $\mu_{\nu_{e}}^{H}=100 \mathrm{MeV}$ in dotted line and $\mu_{\nu_{e}}^{H}=200 \mathrm{MeV}$ in full line). Right panel: the mass-energy density of the hadronic phase at which deconfinement occurs versus the temperature $T$, for the same values of $\mu_{\nu_{e}}^{H}$ given in the left panel (density is given in units of the nuclear saturation density $\rho_{0}$ ). The hadronic phase is described by the GM1 parametrization of the EOS. For the quark phase we adopt the two parameterizations of the NJL model given in Table II. In both figures, if the thermodynamic state of hadronic matter (characterized by $\left\{T^{H}, g^{H}, \mu_{\nu_{e}}^{H}\right\}$ or by $\left\{T^{H}, \rho_{H}, \mu_{\nu_{e}}^{H}\right\}$ ) lies to the left of the curve corresponding to the same $\mu_{\nu_{e}}^{H}$, then the deconfinement transition is not possible. In the right side region of a given curve the preferred phase is deconfined quark matter.
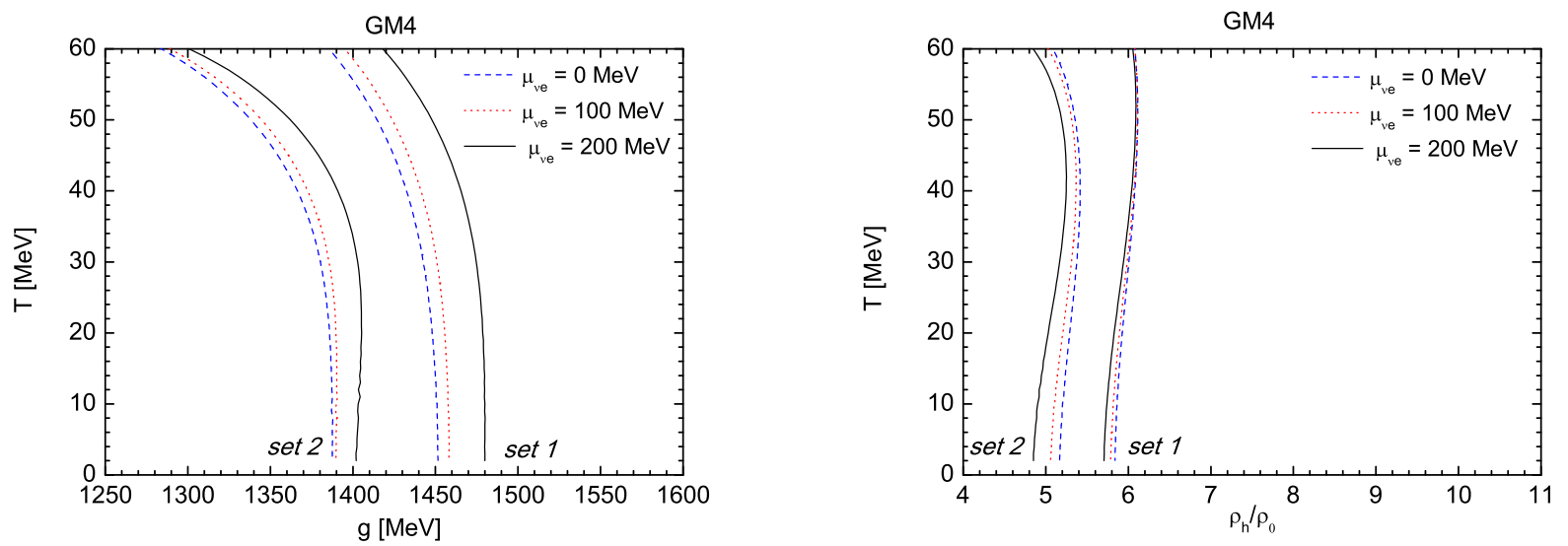

FIG. 2: Same as Fig. 1 but employing the GM4 parametrization of the hadronic equation of state.

\section{CONCLUSIONS}

In this paper we have investigated the role of color superconductivity in the deconfinement transition from hadronic matter to quark matter at finite temperature and in the presence of a trapped neutrino gas. The study presented here is relevant for the first tens of seconds of evolution of newly born protoneutron stars.

In our analysis we used a two phase description where we employed the Nambu-Jona-Lasinio model in the description of quark matter (Sec. III) and a non-linear Walecka model which includes the whole baryon octet, electrons and electron neutrinos in equilibrium under weak interactions in the description of hadronic matter (Sec. II). Deconfinement is assumed to be a first order phase transition and the just deconfined quark phase is assumed to have the same "flavor composition" than the $\beta$-stable hadronic phase from which it has been originated (see [12] and references therein). When color superconductivity is included together with flavor conservation [9], the most likely configuration of the just deconfined phase is 2SC provided the pairing gap is large enough. This just deconfined phase is out of chemical equilibrium under weak interactions and thus it is very short lived but it is a a kind of "activation barrier" 

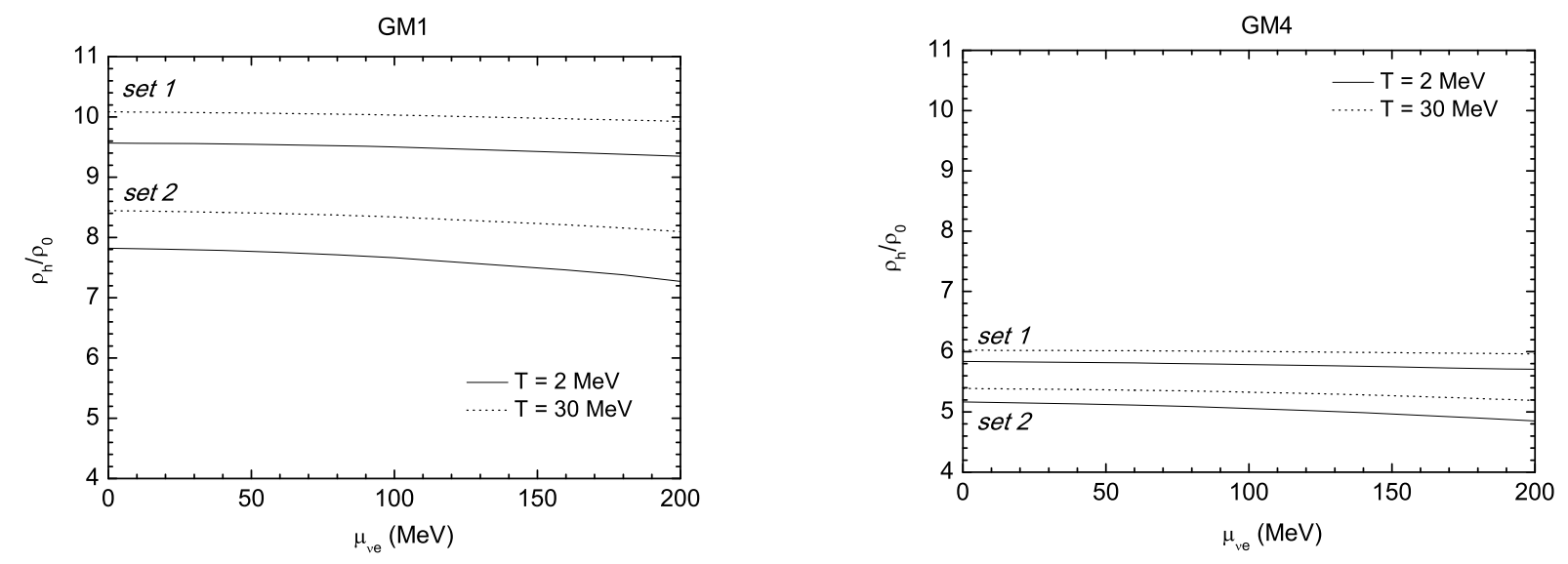

FIG. 3: The mass-energy density of the hadronic phase at which deconfinement occurs as a function of the chemical potential of trapped neutrinos $\mu_{\nu_{e}}^{H}$. Results are given for two temperatures: $T=2 \mathrm{MeV}$ in full line and $T=30 \mathrm{MeV}$ in dashed line. We employed the GM1 (left panel) and the GM4 (right panel) parametrization of the hadronic EOS. Notice that there is a small decrease of the transition density $\rho_{h}$ for large $\mu_{\nu_{e}}^{H}$.

that determines the onset of the deconfinement transition.

The main result of the present paper is that, within the NJL model, deconfinement is more difficult for small neutrino content and it is easier for lower temperatures. This effect is not very large, at least for the here-used parameterizations of the NJL model. At fixed temperature there is a slight increase by less than a $10 \%$ when $\mu_{\nu_{e}}^{H}$ falls from $200 \mathrm{MeV}$ to $0 \mathrm{MeV}$ (see Fig. 3). The effect of cooling is also small; at fixed $\mu_{\nu_{e}}^{H}$ there is a slight decrease of the transition density $\rho_{H}$ by less than a $10 \%$ when the temperature falls from $30 \mathrm{MeV}$ to $2 \mathrm{MeV}$ (see Fig. 3). This is due to the fact that the pairing gap becomes larger as the temperature decreases and therefore the increase of the condensation term favors the transition at low temperatures. Both effects tend to cancel each other as the PNS cools and deleptonizes, resulting a transition density that is roughly constant along the evolution of the protoneutron star.

The here-found behavior is qualitatively opposite to what it was found within the MIT bag model. In fact, previous analysis without including the effect of color superconductivity [4, 5] show that the presence trapped neutrinos pushes up the transition density to values much larger than for neutrino free matter. It was also found in [4, 5] that the transition is easier for larger temperatures. More recent results including the effect of color superconductivity within the MIT Bag model [23] show that the transition density increases with neutrino trapping but (in coincidence with the here found results) the pairing gap favors the transition as the temperature decreases.

In spite of some differences between the results within the NJL and the MIT bag model description of quark matter some general conclusions may be obtained about the effect of color superconductivity in the deconfinement transition. First, when color superconductivity is present the deconfinement density is not so strongly affected by neutrino trapping as it is in the unpaired case. Second, color superconductivity makes the transition easier at lower temperatures and the dependence of the deconfinement density with $T$ is much smaller than in the unpaired case.

During cooling and deleptonization of the protoneutron star the temperature and the chemical potential of trapped neutrinos fall abruptly in a few seconds and there is also some contraction of the whole neutron star. It is interesting to note that although the density increase is not too large, it may be comparatively important for the deconfinement transition because the effects of temperature and neutrino trapping are smoothed by color superconductivity. According to our results the deconfinement transition is favored after substantial cooling and contraction of the protoneutron star but full numerical simulations of protoneutron star evolution are needed in order to determine whether and when the deconfinement conditions are attained.

\section{ACKNOWLEDGEMENTS}

This work was supported in part by CONICET (Argentina) grant \# PIP 6084 and by ANPCyT (Argentina) grant \# PICT07 03-00818. T. A. S. do Carmo acknowledges the financial support received from UFABC (Brazil). G. 
Lugones acknowledges the financial support received from FAPESP (Brazil).

[1] A. Burrows and J. M. Lattimer, Astrophys. J. 307, 178 (1986).

[2] W. Keil and H-Th. Janka, Astron. Astrophys., 296, 145 (1995).

[3] J. A. Pons et al., Astrophys. J. 513, 780, (1999).

[4] G. Lugones and O. G. Benvenuto, Phys. Rev. D 58, 083001 (1998).

[5] O. G. Benvenuto and G. Lugones, Mon. Not. R.A.S. 304, L25 (1999).

[6] K. Iida and K. Sato, Phys. Rev. C 58, 2538 (1998).

[7] M. L. Olesen and J. Madsen, Phys. Rev. D 49, 2698 (1994).

[8] I. Bombaci, I. Parenti, I. Vidaña, Astrophys.J. 614, 314 (2004).

[9] G. Lugones and I. Bombaci, Phys. Rev. D 72, 065021 (2005).

[10] I. Bombaci, G. Lugones, I. Vidaña, Astronomy and Astrophysics 462, 1017 (2007).

[11] I. Bombaci, D. Logoteta, P.K. Panda, C. Providencia, I. Vidana, Phys. Lett. B 680, 448 (2009)

[12] G. Lugones, A. G. Grunfeld, N.N. Scoccola and C. Villavicencio, Phys. Rev. D 80, 045017 (2009)

[13] N. K. Glendenning and S.A. Moszkowski, Phys. Rev. Lett. 67, 2414 (1991).

[14] J.D. Walecka, Ann. Phys. 83, 491 (1974); B.D. Serot and J.D. Walecka, Adv. Nucl. Phys. 16, 1 (1986).

[15] D.P. Menezes and C. Providência, Phys. Rev. C 68, 035804 (2003); A.M.S. Santos and D.P. Menezes, Phys. Rev. C 69, 045803 (2004).

[16] N. K. Glendenning, Astrophys. J. 293, 470 (1985).

[17] M. Huang, P. f. Zhuang and W. q. Chao, Phys. Rev. D 67, 065015 (2003).

[18] S. B. Ruester, V. Werth, M. Buballa, I. A. Shovkovy and D. H. Rischke, Phys. Rev. D 72, 034004 (2005).

[19] D. Blaschke, S. Fredriksson, H. Grigorian, A. M. Oztas and F. Sandin, Phys. Rev. D 72, 065020 (2005).

[20] P. Rehberg, S. P. Klevansky and J. Hufner, Phys. Rev. C 53, 410 (1996).

[21] T. Hatsuda and T. Kunihiro, Phys. Rept. 247, 221 (1994).

[22] M. Buballa, Phys. Rept. 407, 205 (2005).

[23] T. A. S. do Carmo and G. Lugones, to be submitted. 